\title{
EMDR et TCC chez des patients atteints de cancer : étude comparative de leurs effets sur l'ESPT, l'anxiété et la dépression
}

\author{
Liuva Capezzani \\ Service de psychiatrie et Area di Supporto alla Persona, \\ Institut national de cancérologie Regina Elena, Rome, Italie \\ Luca Ostacoli \\ Marco Cavallo \\ Sara Carletto \\ Service de santé mentale, Ecole médicale hospitalière "San Luigi Gonzaga", \\ Université de Turin, ASL TO3, Orbassano, Italie \\ Isabel Fernandez \\ Association EMDR Italie, Bovisio Masciago (MI), Italie \\ Roger Solomon \\ Centre Buffalo pour le trauma et la perte, Buffalo, New York, Etats-Unis \\ Marco Pagani \\ Institut des sciences et technologies cognitives, CNR, Rome, Italie \\ Tonino Cantelmi \\ Service de psychiatrie, Institut national de cancérologie Regina Elena, Rome, Italie
}

\begin{abstract}
Cette étude-pilote teste l'efficacité comparée de la thérapie EMDR (désensibilisation et retraitement par les mouvements oculaires) et de la thérapie cognitive comportementale (TCC) dans le traitement de l'état de stress post-traumatique (ESPT) chez des patients atteints de cancer, dans la phase de suivi de la maladie. Le second objectif de cette étude était d'évaluer si l'EMDR avait un impact différent sur l'ESPT pendant la phase active du traitement ou au cours des étapes de suivi de la maladie. On a assigné aléatoirement vingt-et-un patients en soins de suivi à des groupes d'EMDR ou de TCC, et dix patients en phase active de traitement ont été assignés à un groupe EMDR. Pour évaluer l'ESPT en pré-traitement et à un mois après traitement, on a utilisé l'Impact of Event Scale-Revised (IES-R) et la Clinician-Administered PTSD Scale (CAPS). L'anxiété, la dépression et les symptômes psycho-physiologiques ont également été évalués. Pour les patients en phase de suivi, l'absence d'ESPT après traitement était associée à une probabilité significativement plus grande d'avoir reçu de l'EMDR que de la TCC. L'EMDR était significativement plus efficace que la TCC pour faire décroître les notes à la sous-échelle des symptômes intrusifs de l'IES-R et de la CAPS, mais l'anxiété et la dépression étaient améliorées de façon semblable dans les deux groupes de thérapie. En outre, l'EMDR se montrait aussi efficace dans le traitement actif du cancer que dans la phase de suivi de la maladie.
\end{abstract}

Mots-clés : ESPT ; cancer ; TCC ; EMDR ; psychothérapie

This article originally appeared as Capezzani, L., Ostacoli, L., Cavallo, M., Carletto, S., Fernandez, I., Solomon, R., Pagani, M., \& Cantelmi, T. (2013). EMDR and CBT for Cancer Patients: Comparative Study of Effects on PTSD, Anxiety, and Depression. Journal of EMDR Practice and Research, 7(3), 134-143. Translated by François Mousnier-Lompré. 
es études sur le stress ou les symptômes traumatiques des patients atteints de cancer ne sont pas nouvelles (Andersen, Kiecolt-Glaser, \& Glaser, 1994 ; Butler, Koopman, Classen, \& Spiegel, 1999 ; Mehnert \& Koch, 2007). Cependant, depuis quelques années, les recherches se concentrent sur les types de stress propres à ces patients, et de nombreuses études ont avancé que cette population, face au diagnostic du cancer et aux difficultés de vivre avec la maladie, éprouve des stress tout à fait analogues à ce qu'éprouvent les victimes de crimes violents ou de catastrophes naturelles (Cordova, Studts, Hann, Jacobsen, \& Andrykowski, 2000 ; Jackson et al., 2007). L'état de stress post-traumatique (ESPT) qu'on associe couramment aux rescapés de situations traumatiques de cet ordre est actuellement en cours de documentation chez les patients atteints de cancer (Bruce, 2006 ; DuHamel et al., 2004).

L'ESPT est un trouble caractérisé par l'apparition de certains symptômes après un événement émotionnellement stressant, avec une mort réelle ou une menace de mort, une blessure grave ou un danger pour soi-même ou d'autres (National Cancer Institute, 2012b). Cependant, il est important de remarquer que jusqu'en 1987, la troisième édition révisée du Diagnostic and Statistical Manual of Mental Disorders (DSM-III-R ; American Psychiatric Association, 1987) excluait du champ de l'ESPT les patients souffrant de maladies comme le cancer. Grâce à une révision de texte dans la quatrième édition du DSM (DSM-IV-TR), publiée en 2000 (American Psychiatric Association, 2000), les critères diagnostiques de l'ESPT comprennent expressément "le fait de recevoir un diagnostic de maladie mortelle" comme un exemple d'événement traumatisant, et on peut dorénavant considérer comme à risque d'ESPT et évaluer pour ce trouble les personnes ayant eu un cancer (National Cancer Institute, 2012a).

Plusieurs études de l'ESPT, portant sur toutes sortes de cancers (mélanomes, maladie de Hodgkin, cancer du sein et cancers mixtes), ont eu lieu dans cette population, mais elles n'étaient pas homogènes dans leurs évaluations de l'ESPT ; certaines évaluaient le patient par rapport au syndrome d'ESPT complet (c'est-à-dire remplissant tous les critères du DSM-IV) ou seulement par rapport à certains symptômes liés à l'ESPT (p. ex., les pensées intrusives mesurées par l'Impact of Event Scale-Revised ; IES-R ; National Cancer Institute, 2012a). Il est important de noter que l'ESPT est difficile à diagnostiquer dans n'importe quelle population, pour plusieurs raisons : d'abord, parce qu'on peut le confondre avec de nombreux autres troubles psychologiques, et ensuite parce que les symptômes peuvent apparaître de façon retardée dans le temps, dans certains cas bien des années après l'expérience traumatique. Chez les patients atteints de cancer, dans une phase particulière de la maladie, les symptômes peuvent rester juste sous la surface, et empêcher, même s'ils sont traités, une rémission complète. Une recherche récente, entreprise par le Duke Cancer Institute, est l'une des rares études, à ce jour, qui fournisse des informations valables après une période importante de suivi ; elle documente l'ESPT chez des patients atteints de lymphome non-hodgkinien sur une période moyenne de suivi de 12 ans et 9 mois (Smith et al., 2011). En fait, elle a montré que l'ESPT s'aggrave avec les années. Il n'a pas été développé, pour cette population, de stratégie psychothérapeutique unique pour l'ESPT; cependant, la littérature, sur le sujet de l'ESPT, est riche d'exemples de nombreuses formes de psychothérapie efficaces, parmi lesquelles la thérapie cognitive-comportementale (TCC), comme l'ont montré des études internationales (Foa, Keane, Friedman, \& Cohen, 2008 ; Rothbaum, Astin, \& Marsteller, 2005 ; Taylor et al., 2003) et l'EMDR (désensibilisation et retraitement par les mouvements oculaires ; Shapiro, 1995, 2001). La thérapie EMDR a été reconnue par la Société internationale pour l'étude du Stress Traumatique (International Society for Traumatic Stress Studies; Chemtob, Tolin, van der Kolk \& Pitman, 2000) et, en 2001, elle a été reconnue comme une forme de thérapie efficace de l'ESPT (classification A/B) par le Ministère britannique de la Santé (Bisson et al., 2007 ; Onofri, 2012). On l'utilise dans le monde entier depuis 1990, et elle s'est avérée efficace dans toutes sortes de formes de stress et de troubles d'origine traumatique, dont l'ESPT.

L'EMDR a traité efficacement des patients souffrant de maladies variées, comme la douleur chronique (Grant \& Threlfo, 2002 ; Schneider, Hofmann, Rost \& Shapiro, 2008), la fibromyalgie (Friedberg, 2004), et l'infarctus du myocarde (Arabia, Manca \& Solomon, 2011). En particulier, une étude pilote récente a fourni une preuve préliminaire que l'EMDR était plus efficace que la thérapie par exposition imaginaire dans le traitement de patients rescapés d'un accident cardiaque mettant leur vie en jeu (Arabia et al., 2011). Notre étude pilote est le premier projet structuré de recherche en Italie qui étudie la TCC et l'EMDR chez des patients atteints de cancer traités à l'Institut national de cancérologie Regina Elena de Rome.

Dans la littérature de psycho-oncologie concernant la réaction de l'individu à sa maladie, on trouve deux hypothèses prévalentes : la première considère que le malade atteint de cancer est connecté à une série de crises qui se produisent au cours de la maladie et 
impliquent des changements dans l'écosystème environnemental du patient (Morasso, 2002). La seconde se centre davantage sur l'individu et le considère comme vulnérable ou prédisposé à des troubles psychopathologiques (Morasso, 2002). En se centrant sur une "maladie de crises", on risque de n'avoir qu'une appréciation superficielle de la maladie psycho-oncologique, et de négliger l'impact traumatique de ce vécu ainsi que le mal-être psychologique lié au cancer, qui est tellement vécu comme "traumatique" qu'on peut arriver à un diagnostic d'ESPT - et cette perturbation est au cour de la psychopathologie de la personne.

Le cancer est objectivement traumatisant (Castrogiovanni \& Traverso, 2006). Les classifications suivantes, tirées du DSM-IV-TR, montrent que la communauté scientifique reconnaît le puissant impact traumatique de cette maladie sur l'individu.

- Le cancer produit un sentiment de mise en danger de la vie de la personne, de la qualité de vie et de l'intégrité psychologique et physique de la personne et des autres, y compris sa famille (critère A1 de l'ESPT, DSM-IV-TR ; American Psychiatric Association, 2000).

- Il crée une impression écrasante de vulnérabilité, de perte de contrôle et d'impuissance (critère A2 de l'ESPT, DSM-IV-TR ; American Psychiatric Association, 2000).

- La personne présente de fortes réactions émotionnelles avec des pensées intrusives, un évitement des comportements quotidiens, une hyperactivation, à la fois sous forme aiguë et sous forme chronique, qui entravent la capacité normale de fonctionnement du sujet (critères B, C, D, E et F pour l'ESPT, DSM-IV-TR ; American Psychiatric Association, 2000).

Comme le précise le DSM-IV-TR (American Psychiatric Association, 2000), pour ce qui concerne le critère E de l'ESPT, les symptômes doivent durer au moins un mois, ce qui peut être le cas après l'annonce du diagnostic d'un cancer.

\section{Objectifs}

Le premier objectif de cette étude pilote était d'évaluer l'efficacité comparée de l'EMDR et de la TCC chez des patients atteints de cancer souffrant d'ESPT dans la phase de suivi de la maladie. Nous souhaitions évaluer l'efficacité de l'EMDR et de la TCC sur des mesures particulières d'ESPT, ainsi que sur les symptômes liés à l'ESPT : anxiété, dépression et réactions psychophysiologiques. Le second objectif était d'établir si l'EMDR avait un impact différent sur l'ESPT et ses symptômes, à deux phases différentes de la maladie (traitement actif du cancer/suivi post-traitement), pour aborder la question de ses éventuels bénéfices pour les patients atteints de cancer dans les phases antérieures de leur traitement médical.

\section{Méthodes}

\section{Participants}

Trente-et-un patients atteints de différents types de cancer (cancer du sein, du côlon, de l'utérus, de la thyroïde, du poumon, de l'estomac, et mélanome) ont été recrutés entre mai 2010 et juin 2012 dans les services de chirurgie digestive, de chirurgie thoracique et d'oncologie médicale (Service B) de l'Institut national de cancérologie Regina Elena de Rome. Le recrutement a été fait en incluant toutes les prises en charge adressées à la clinique psychiatrique par les unités oncologiques, qui satisfaisaient au diagnostic clinique d'ESPT (31 patients sur 623 , soit $4,97 \%$ ). Les 31 patients ont donné leur accord pour participer à l'étude.

Les critères d'inclusion étaient les suivants : a) remplir les critères diagnostiques ESPT du DSM-IV et b) ne pas avoir de traitement psychopharmacologique en cours.

Les critères d'exclusion concernaient : a) les patients déjà en psychothérapie et b) les patients ayant des troubles psychopathologiques préexistants au diagnostic de cancer.

Les patients ont été placés dans l'une ou l'autre étude en fonction de l'étape de la maladie où ils se trouvaient : dix étaient en phase active de traitement $\mathrm{du}$ cancer et vingt-et-un en phase de suivi posttraitement. Ces vingt-et-un patients ont été assignés au hasard à un groupe TCC ou à un groupe EMDR. Ceux qui étaient en phase de traitement $(\mathrm{n}=10)$ ont été assignés seulement à l'étude sur le traitement EMDR. Au total, on a trois groupes de patients : un groupe de patients en phase de traitement faisant seulement de l'EMDR ( $\mathrm{n}=10)$, un groupe de patients en phase de suivi faisant de l'EMDR $(\mathrm{n}=11)$ et un autre groupe de patients en phase de suivi faisant de la TCC $(\mathrm{n}=10)$.

\section{Mesures}

Tous les questionnaires utilisés pour l'évaluation des participants à l'étude sont auto-administrés, saufle ClinicalAdministered PTSD Scale (CAPS), qui a été administré par un examinateur indépendant, en aveugle.

Le Clinician-Administered PTSD Scale (CAPS). Le CAPS (Blake et al., 1995), sous sa forme Current and Lifetime Diagnostic Version (version DX) est un 
entretien clinique semi-structuré fondé sur le DSM-IV$T R$, qui est la référence pour l'évaluation de l'ESPT (Foa \& Tolin, 2000; Weathers, Keane, \& Davidson, 2001). Sa structure correspond aux critères du DSM$I V$, avec une évaluation des symptômes B (intrusion), $\mathrm{C}$ (évitement) et $\mathrm{D}$ (hyperactivation) à la fois sur le plan de leur fréquence et de leur intensité ; les deux notes sont ensuite additionnées pour obtenir les évaluations de sévérité. Des questions supplémentaires évaluent les critères $\mathrm{A}, \mathrm{E}$ et $\mathrm{F}$.

L'Impact of Event Scale-Revised (IES-R). L'IES$\mathrm{R}$ (Weiss \& Marmar, 1997) est un questionnaire en 22 questions avec trois sous-échelles (intrusion, évitement, hyperactivation) qui évalue la détresse subjective causée par les événements traumatiques. On demande au patient d'identifier un événement stressant particulier de sa vie, puis d'indiquer à quel point il a été perturbé ou ennuyé par chaque "difficulté” listée au cours des sept jours avant le test.

The Psychophysiological Questionnaire-Brief Version (QPF-R). Le QPF-R (Pancheri, Chiari, \& Michielin, 1985) a été utilisé pour évaluer les réactions psycho-physiologiques. Il comprend trente items, sur une échelle de Likert (de 0 à 4 ) qui se rapporte aux symptômes somatiques sans base organique démontrable.

Le State-Trait Anxiety Inventory (STAI-Y). On se sert du STAI-Y (Spielberger, Gorsuch, \& Lushene, 1970) pour évaluer l'anxiété-état (STAI-1) et l'anxiété-trait (STAI-2) du sujet. Il comprend quarante questions : vingt pour l'anxiété-état et vingt pour l'anxiété-trait. Chaque item est évalué sur une échelle de Likert, de 0 à 4 .

L'Inventaire de dépression de Beck (Depression Inventory-II, BDI). Le BDI (Beck \& Steer, 1993) est un instrument auto-administré en 21 items évaluant la présence et la gravité de symptômes correspondant aux critères du DSM-IV.

\section{Procédure}

Evaluation. Les évaluations ont été menées avant le traitement et un mois après la fin des séances de traitement.

Pour le premier entretien, les patients arrivaient au centre adressés par un médecin hospitalier ou par un médecin généraliste. Quand la psychologue (L. C.) repérait des éléments cliniques pouvant évoquer un diagnostic d'ESPT, elle invitait le patient à rencontrer un évaluateur indépendant, travaillant en aveugle, qui utilisait alors le CAPS pour explorer un éventuel ESPT. Les évaluations comprenaient un entretien clinique avec le patient au sujet de son passé médical, entretien mené par le clinicien en charge du patient au cours du pré-traitement et du post-traitement. Ensuite, les patients chez qui le diagnostic d'ESPT était confirmé se voyaient demander de remplir les autres questionnaires psychologiques auto-administrés ; ces questionnaires leur étaient remis par un évaluateur indépendant, qui était également à leur disposition pour répondre à leurs éventuelles questions sur la compilation des questionnaires. Puis on leur proposait de prendre part à l'étude. S'ils étaient d'accord, ils discutaient du formulaire de consentement éclairé et le signaient.

Ceux qui étaient dans la phase de suivi de la maladie étaient aléatoirement assignés en thérapie EMDR ou en thérapie TCC. S'ils étaient dans la phase active de traitement de la maladie, on les assignait seulement en thérapie EMDR.

Traitement. Tous les participants, quel que soit le type de thérapie reçue ou la phase de la maladie où ils se trouvaient, recevaient huit séances de thérapie hebdomadaire. On conseillait à tous les patients présentant encore des symptômes au-delà d'un mois après la fin de la thérapie de poursuivre le travail avec leur thérapeute, même si l'étude était finie.

EMDR. Le protocole standard EMDR (Shapiro, 2001) en huit étapes a été administré, avec pour objectifs :

1. Stabilisation par la psychoéducation (sur l'adaptation émotionnelle face au cancer, les caractéristiques de l'ESPT et la thérapie EMDR) et "l'installation de ressources" (Shapiro, 2001) avec la technique du "lieu sûr" (Shapiro, 2001) pour stabiliser le patient et le préparer à la thérapie.

2. Identification et retraitement de souvenirs perturbants liés au cancer dans les trois dimensions de l'EMDR : événements passés et présents, identifiés lors du recueil de l'histoire de la personne et à l'aide de floatbacks (p. ex. l'annonce du diagnostic ou d'une récidive, l'annonce de complications dans le traitement, les effets secondaires du traitement) et modèles pour le futur, pour gérer les préocupations et les peurs (p. ex. la crainte d'une détérioration du fonctionnement physique, la peur de l'avenir). On a suivi la totalité des huit phases de l'EMDR (Shapiro, 2001) et la thérapie a été uniquement centrée sur le cancer, à l'exclusion de tout événement traumatique antérieur.

3. Intégration: la réduction de l'anxiété et le développement de ressources ont été intégrés dans la vie quotidienne des patients pour améliorer leur adaptation à leur situation de rescapés de la maladie. 
Toutes les thérapies EMDR ont été menées par un thérapeute ayant dix ans d'expérience clinique en EMDR.

TCC. On trouvera ci-après les techniques et les approches thérapeutiques utilisées en fonction des symptômes d'ESPT qui étaient le plus souvent rapportés par chaque patient, et en fonction des étapes de la psychothérapie. Voici les objectifs qu'on s'était fixés :

1. Stabiliser les symptômes initiaux du patient. Pour l'hyperactivation : psychoéducation, REI (Rational Emotive Imagery ; Ellis, 1994), une technique de visualisation guidée, une exposition graduelle ou prolongée au stresseur in vivo ou en imagination, et des techniques de relaxation progressive avec des consignes portant sur la respiration diaphragmatique pour traiter l'insomnie. Pour l'hypoactivation : psychoéducation, instructions de travail personnel visant à définir, reconnaître et activer des ressources somatiques (p. ex. un programme structuré d'activité physique ; Beck, Rush, Shaw \& Emery, 1979).

2. Pour les flash-backs et les pensées intrusives : techniques de déplacement de l'attention.

3. Pour les comportements d'évitement ou de fuite : désensibilisation systématique et exposition graduelle par exposition imaginaire ou in vivo (personnes ou objets).

4. Pour la restructuration cognitive ou les pensées cognitives négatives en rapport avec le vécu traumatique : formes A, B, C, D, E de la Rational Emotive Behavior Therapy (REBT; Ellis, 1994) et dialogue socratique.

5. Pour le contrôle des fluctuations psycho-physiologiques et le maintien des nouveaux patterns comportementaux : travail personnel et journal de bord.

6. Pour l'observance du traitement : techniques de contrôle et redéfinition des alliances thérapeutiques.

Toutes les thérapies TCC ont été menées par le thérapeute qui menait aussi les thérapies EMDR, qui a par ailleurs douze ans d'expérience de clinicien TCC.

\section{Analyses statistiques}

Les données ont été traitées et analysées à l'aide du Statistical Package for Social Sciences (SPSS) version 17.0 (Chicago, IL, USA).

Les différences d'origine des groupes ont été évaluées en utilisant une analyse de la variance à un facteur (one-way ANOVA) pour comparer les trois groupes sur le plan des mesures continues, et le test exact de Fisher pour les comparer sur celui des mesures catégorielles.
Le test exact de Fisher a également été utilisé pour évaluer le rapport entre le groupe thérapeutique (EMDR ou TCC) et le diagnostic d'ESPT au moment T1.

Le modèle linéaire généralisé (Generalized linear model, GLM) de l'analyse de variance multivariée par mesures répétées (RM-MANOVA) a été utilisé pour analyser les effets et les interactions essentiels pré- et post-intervention, à la fois entre les groupes EMDR et TCC et dans chacun d'eux, au cours de la phase de suivi de la maladie. Des comparaisons par paires entre les groupes ont été faites par contraste simple et rapportées comme différence moyenne avec l'intervalle de confiance corrigé (IC 95\%) de Sidak pour les comparaisons multiples.

Des analyses logistiques simples de régression ont été calculées en prenant la présence d'un ESPT après le traitement comme variable dépendant dichotomique, et en considérant isolément comme variables indépendantes l'âge, le sexe, le type de thérapie (EMDR/TCC), et les notes des variables cliniques du départ (QPF-R, STAI-1, STAI-2, BDI-II, IES-R total, critères $B, C$ et $D$ du CAPS) pour les patients qui se trouvaient dans la phase de suivi du cancer.

Comme résultat secondaire, on a utilisé une RMMANOVA pour analyser les effets et les interactions essentiels pré- et post-intervention, à la fois entre les groupes selon les différentes phases du traitement anticancéreux (traitement actif ou suivi) et à l'intérieur de chacun d'eux, pour évaluer si la thérapie EMDR a un impact différent, suivant l'étape de la maladie où on la fait intervenir.

Enfin, un $p<0,05$ a été considéré comme statistiquement significatif dans toutes les analyses.

\section{Résultats}

31 patients ont été incorporés à l'étude : 10 dans une phase active de traitement du cancer, tous traités en EMDR, et les 21 autres dans une phase de suivi médical. De ce second groupe, 11 ont été placés aléatoirement en thérapie EMDR et 10 en thérapie TCC. Aucun patient n'a quitté la thérapie en cours de route. Les résultats ont montré que les différents types de cancer (cancer du sein, du côlon, de l'utérus, de la thyroïde, du poumon, de l'estomac, et mélanome) étaient répartis de façon équilibrée dans les trois groupes.

Comparaison entre EMDR et TCC dans la phase de suivi du cancer

On l'a dit, il y avait 21 patients en phase de suivi, parmi lesquels 11 ont été placés aléatoirement en thérapie EMDR (tous étaient des femmes) et 10 en thérapie 
TABLEAU 1. Données cliniques des participants en phase de suivi du cancer

\begin{tabular}{|c|c|c|c|c|c|}
\hline & \multicolumn{2}{|c|}{ Pré-traitement } & \multicolumn{2}{|c|}{ Post-traitement } & \multirow[b]{2}{*}{ Sig. } \\
\hline & $\begin{array}{c}\text { TCC } \\
(\mathrm{N}=10)\end{array}$ & $\begin{array}{c}\text { EMDR } \\
(\mathrm{N}=11)\end{array}$ & $\begin{array}{c}\text { TCC } \\
(\mathrm{N}+10)\end{array}$ & $\begin{array}{c}\text { EMDR } \\
(\mathrm{N}=11)\end{array}$ & \\
\hline QPF-R & $61,60(15,71)$ & $57,45(13,55)$ & $54,50(13,24)$ & $48,45(12,18)$ & $\star$ \\
\hline STAI-1 & $45,40(4,95)$ & $44,73(5,42)$ & $43,90(5,55)$ & $40,00(3,41)$ & * \\
\hline STAI-2 & $46,50(5,34)$ & $45,82(6,15)$ & $43,80(4,10)$ & $43,55(5,70)$ & \\
\hline BDI-II & $26,30(8,73)$ & $25,73(10,89)$ & $20,10(9,24)$ & $14,45(9,30)$ & $\star$ \\
\hline IES-R total & $54,70(10,62)$ & $50,91(9,45)$ & $46,60(14,13)$ & $20,55(17,85)$ & ${ }^{\star}, \S$ \\
\hline CAPS critère B & $20,90(7,71)$ & $19,55(8,15)$ & $15,30(5,87)$ & $6,18(6,95)$ & ${ }^{\star}, \S$ \\
\hline CAPS critère C & $30,30(8,13)$ & $28,36(12,19)$ & $20,50(7,59)$ & $10,45(7,54)$ & $\star$ \\
\hline CAPS critère D & $27,60(6,22)$ & $24,00(8,15)$ & $16,20(9,16)$ & $9,91(5,61)$ & $\star$ \\
\hline
\end{tabular}

Note. Ces données sont des moyennes (écarts-types). QPF-R = Psychophysiological Questionnaire-Brief Version ; STAI-1 = StateTrait Anxiety Inventory—anxiété-état ; STAI-2 = State-Trait Anxiety Inventory—anxiété-trait ; BDI-II = Inventaire de dépression de Beck-II $;$ IES-R total = Impact of Event Scale-Revised—note totale $;$ CAPS critère B = Clinician-Administered PTSD Scale—symptômes intrusifs ; CAPS critère C = Clinician-Administered PTSD Scale—symptômes d'évitement ; CAPS critère D = Clinician-Administered PTSD Scale—symptômes d'hyperactivation.

* : effet significatif pré-post thérapie, sans considération du type de thérapie (TCC ou EMDR)

$\S$ : effets d'interaction significatifs en fonction du groupe (TCC ou EMDR) et en fonction de la période (pré-traitement ou post-traitement).

TCC (huit femmes et deux hommes). L'âge moyen des patients était similaire dans les deux groupes $(52,70 ; \sigma=8,68$ pour la TCC et 50,$82 ; \sigma=7,64$ pour l'EMDR).

Il n'y avait pas de différences de variables cliniques entre les deux groupes au départ (Tableau 1).

Nous avons évalué si les deux différentes formes de thérapie (EMDR ou TCC) administrées aux patients pendant la phase de suivi du cancer avaient une incidence différente sur les variables psychologiques auxquelles l'étude s'intéressait.

Pour ce faire, on a réalisé une MANOVA par mesures répétées sur les notes cliniques avant et après la thérapie (QPF-R, STAI-1, STAI-2, BDI-II, IES-R Total, CAPS critères $B, C$, et $D$ ) en comparant les effets de groupe et de période et les interactions entre groupe et période.

Cette RM-MANOVA a montré un effet principal significatif pré-post $(\mathrm{F}[8,12]=13,547, p<0,001$; $\left.\eta_{\mathrm{p}}^{2}=0,00\right)$ et une interaction significative entre les mesures pré-post et la forme de thérapie ( $\mathrm{F}[8$, $\left.12]=4,855, p=0,007 ; \eta_{\mathrm{p}}^{2}=0,764\right)$.

Des effets significatifs de la période ont été trouvés dans les deux groupes pour toutes les variables, excepté pour la STAI-2 (l’anxiété-trait), ce qui indique que les notes moyennes des participants se sont améliorées entre la période 1 (pré-intervention) et la période 2 (post-intervention; cf. Tableau 1).

Des effets de l'interaction groupe/période ont été trouvés pour les notes totales à l'IES-R (F[1,
19] $=14,041, p<0,001 ; \mathrm{cf}$. Figure 1) et pour les notes du critère $\mathrm{B}$ du CAPS $(\mathrm{F}[1,19]=7,584, p=0,013 ; \mathrm{cf}$. Tableau 1 et Figure 2).

Aucune interaction groupe/période n'a été trouvée pour les QPF-R, STAI-1, BDI-II, IES-R, CAPS-C, et CAPS-D, ce qui indique que les changements sur ces mesures étaient similaires pour les deux groupes de thérapie.

On a conduit les analyses post hoc prévues sur les effets simples pré-post par comparaisons GLM par paires en utilisant l'ajustement de Sidak pour les comparaisons multiples (cf. Figure 1). Les résultats ont montré que la note totale à l'IES-R, au post-traitement $(\mathrm{m}=20,56$; erreur type $=4,880)$, était significativement plus basse que la note au pré-traitement pour le groupe EMDR ( $\mathrm{m}=50,91$; erreur type $=3,020)$, différence moyenne $=-30,364$ (IC 95\% $[-38,945$, $-21,782]), p<0,001$. Pour les participants qui avaient suivi le traitement TCC, il n'y avait pas de différence entre les notes post-traitement $(\mathrm{m}=46,60$, erreur type $=5,12)$ et les notes pré-traitement $(\mathrm{m}=17,73$, erreur type $=1,497$ ), différence moyenne $=-8,100$ (IC $95 \%[-17,100,0,900]), p=0,075$. Cela indique que les améliorations au fil du temps étaient significativement plus importantes dans le groupe de thérapie EMDR que dans le groupe TCC (cf. Figure 1). L'analyse des effets simples révèle aussi une différence significative entre les notes post-traitement EMDR et TCC à l'IES-R : celles du groupe EMDR $(\mathrm{m}=20,55$, erreur type $=4,88$ ) sont significativement plus 


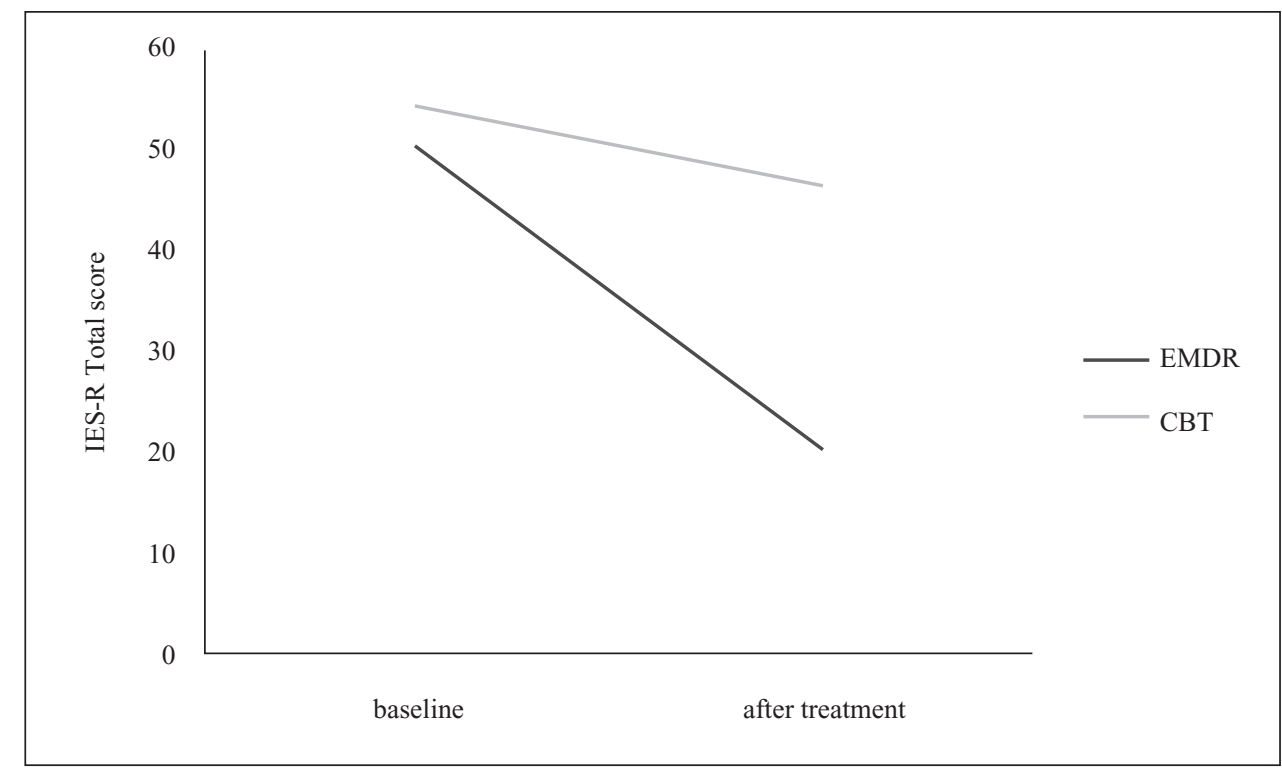

FIGURE 1. Interaction entre la période et le forme de thérapie pour la note totale à l'IES-R.

Note. IES-R Total score $=$ note totale IES-R $;$ CBT $=$ TCC $;$ baseline $=$ au départ $;$ after treatment $=$ après la thérapie.

basses que celles du groupe TCC $(\mathrm{m}=46,60$, erreur type $=5,12)$, différence moyenne $=-26,055($ IC $95 \%$ $[-40,865,-11,244]), p=0,002$.

On a mené également les analyses post hoc prévues sur les effets simples pour la sous-échelle "intrusion" du CAPS (cf. Figure 2). L'analyse de ces effets simples a montré que la note au critère $B$, au post-traitement $(\mathrm{m}=6,18$, erreur type $=1,95$ ), était significativement plus basse que la note pré-traitement pour le groupe $\operatorname{EMDR}(\mathrm{m}=19,56$, erreur type $=2,40)$, différence moyenne $=-13,364$ (IC
$95 \%[217,435,29,292]), p<0,001$. Il y avait également une différence entre les notes au post-traitement $(\mathrm{m}=$ $15,30$, erreur type $=2,04)$ et au pré-traitement $(\mathrm{m}=$ 20,90 , erreur type $=2,51$ ) pour les participants au groupe TCC, différence moyenne $=-5,600$ (IC 95\% $[29,870$, 21,330]), $p=0,013$.

Les deux groupes avaient certes connu une amélioration des symptômes intrusifs, mais la comparaison entre les notes au critère $\mathrm{B}$ du CAPS au post-traitement montrait des notes significativement plus

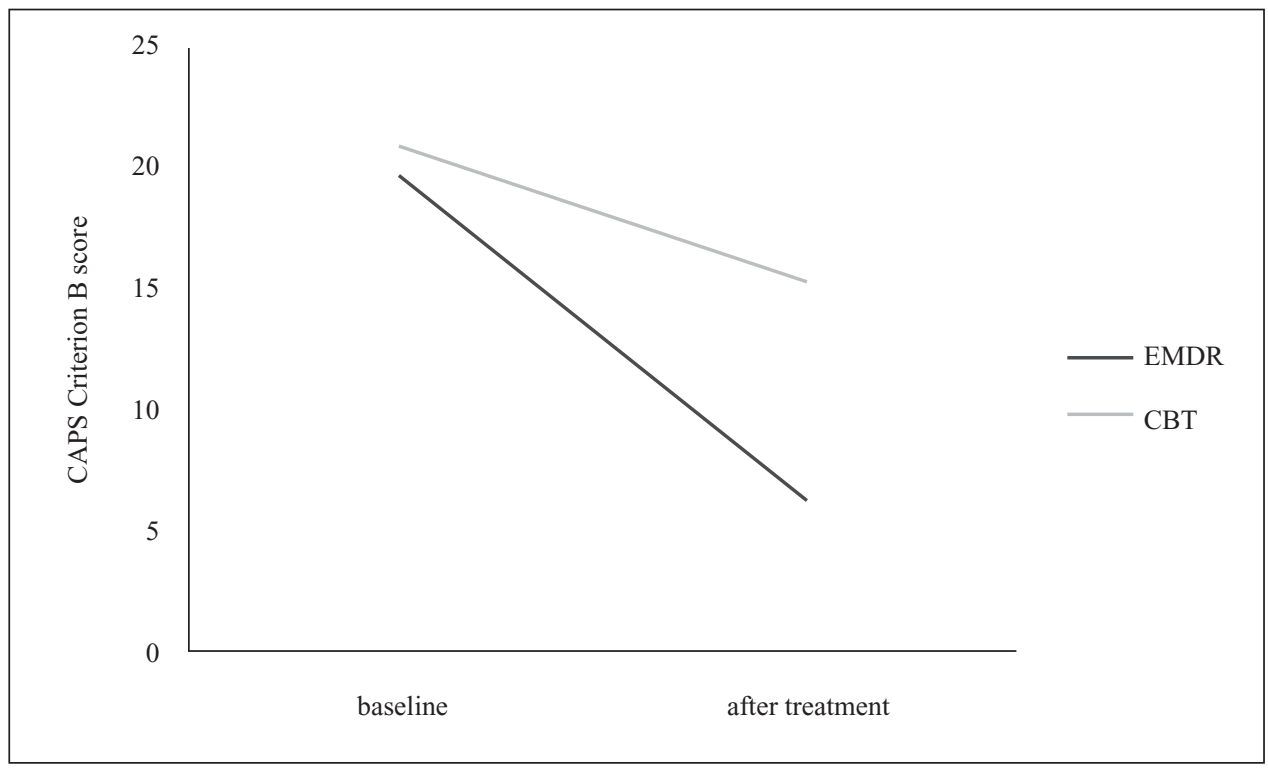

FIGURE 2. Interaction entre la période et la forme de thérapie pour la note au critère $B$ du CAPS.

Note. CAPS Criterion B score $=$ note au critère $\mathrm{B}$ du CAPS $; \mathrm{CBT}=\mathrm{TCC} ;$ baseline $=$ au départ $;$ after treatment $=$ après la thérapie. 
TABLEAU 2. Variables cliniques des groupes traités en EMDR (pré- et post-traitement)

\begin{tabular}{|c|c|c|c|c|c|}
\hline & \multicolumn{2}{|c|}{ Pré-traitement } & \multicolumn{2}{|c|}{ Post-traitement } & \multirow[b]{2}{*}{ Sig. } \\
\hline & $\begin{array}{c}\text { TCC } \\
(\mathrm{N}=10)\end{array}$ & $\begin{array}{c}\text { EMDR } \\
(\mathrm{N}=11)\end{array}$ & $\begin{array}{c}\text { TCC } \\
(\mathrm{N}+10)\end{array}$ & $\begin{array}{c}\text { EMDR } \\
(\mathrm{N}=11)\end{array}$ & \\
\hline QPF-R & $58,50(9,70)$ & $57,45(13,55)$ & $48,30(9,65)$ & $48,45(12,18)$ & $\star$ \\
\hline STAI-1 & $43,70(3,37)$ & $44,73(5,42)$ & $42,70(3,50)$ & $40,00(3,41)$ & $\star$ \\
\hline STAI-2 & $46,10(5,65)$ & $45,82(6,15)$ & $43,30(4,55)$ & $43,55(5,70)$ & \\
\hline BDI-II & $27,00(7,70)$ & $25,73(10,89)$ & $15,50(8,33)$ & $14,45(9,30)$ & $\star$ \\
\hline IES-R total & $48,50(14,74)$ & $50,91(9,45)$ & $28,60(9,38)$ & $20,55(17,85)$ & $\star$ \\
\hline CAPS critère B & $20,70(6,82)$ & $19,55(8,15)$ & $6,20(3,08)$ & $6,18(6,95)$ & $\star$ \\
\hline CAPS critère C & $22,50(4,09)$ & $28,36(12,19)$ & $7,40(3,89)$ & $10,45(7,54)$ & $\star$ \\
\hline CAPS critère D & $19,90(9,25)$ & $24,00(8,15)$ & $6,60(4,22)$ & $9,91(5,61)$ & $\star$ \\
\hline
\end{tabular}

Note. Ces données sont des moyennes (écarts-types) ou $N(\%)$. QPF-R = Psychophysiological Questionnaire - Brief Version ; STAI-1 = State-Trait Anxiety Inventory — anxiété-état ; STAI-2 = State-Trait Anxiety Inventory — anxiété-trait ; BDI-II = Inventaire de dépression de Beck-II ; IES-R total = Impact of Event Scale-Revised — note totale ; CAPS critère B = Clinician-Administered PTSD Scale — symptômes intrusifs ; CAPS critère $\mathrm{C}=$ Clinician-Administered PTSD Scale — symptômes d'évitement ; CAPS critère $\mathrm{D}=$ Clinician-Administered PTSD Scale — symptômes d'hyperactivation.

* : effet significatif pré-post thérapie, sans considération de la phase de la maladie (traitement actif ou suivi).

basses $(\mathrm{m}=6,18$, erreur type $=1,95)$ dans le groupe EMDR que dans le groupe TCC $(\mathrm{m}=15,30$, erreur type $=5,04)$, différence moyenne $=-9,118($ IC $95 \%$ $[-15,029,-3,207]), p=0,004$.

De plus, on a mené une analyse de régression logistique binaire pour détecter l'influence éventuelle du type de thérapie (EMDR ou TCC) et l'influence des variables cliniques sur la présence de l'ESPT après les huit séances de thérapie. On a pu montrer que la présence d'ESPT après la thérapie était significativement associée au type de thérapie seulement (EMDR ou TCC $; R^{2}=0,71 ;$ OR $=0,011$, IC $95 \%[0,001,-0,205]$; $p=0,002)$. L'influence des autres variables démographiques et cliniques n'atteignait pas un niveau statistiquement signifiant. L'absence d'ESPT après la thérapie était associée à une probabilité supérieure d'avoir entrepris un traitement EMDR. Plus précisément, 10 des 11 sujets traités par l'EMDR n'avaient plus d'ESPT après leur thérapie, alors que 9 sur 10 des patients traités par TCC conservaient un diagnostic d'ESPT à l'évaluation post-intervention (Test exact de Fisher ; $\left.p<0,001, \eta_{\mathrm{p}}{ }^{2}=0,809\right)$.

Efficacité de la thérapie EMDR aux deux étapes de la maladie (traitement actif du cancer ou phase de suivi)

En deuxième objectif, nous avons évalué si le traitement EMDR avait un impact différent selon que les patients étaient en phase de traitement médical actif ou en phase de suivi. Les 21 ont été divisés comme suit : dix d'entre eux (neuf femmes et un homme) étaient en phase active de traitement de leur cancer, les onze autres (toutes des femmes) en phase de suivi. L'âge moyen des patients était comparable dans les deux groupes $(53,40, \sigma=8,59$ pour les patients en phase active de traitement et 50,82, $\sigma=7,64$ pour les patients en phase de suivi). Il n'y avait pas de différence dans les variables cliniques entre les deux groupes au départ (cf. Tableau 2).

La RM-MANOVA a montré un effet principal pré-post significatif $(\mathrm{F}[8,12]=22,900, p<0,001$; $\left.\eta_{p}{ }^{2}=0,939\right)$, alors qu'aucune interaction significative n'était trouvée entre les mesures pré-post et le fait de se trouver dans l'une ou l'autre des phases de traitement du cancer (traitement actif ou suivi) $\left(\mathrm{F}[8,12]=0,885, p=0,555 ; \eta_{\mathrm{p}}{ }^{2}=0,371\right)$. Il a été trouvé des effets significatifs liés au temps dans les deux groupes, pour toutes les variables sauf pour STAI-2 (anxiété-trait), ce qui montrait que, indépendamment de la phase de la maladie, les notes s'amélioraient du moment 0 (pré-intervention) au moment 1 (post-intervention). Par conséquent, on peut dire que la thérapie EMDR est efficace quelle que soit la phase de la maladie.

Pratiquement plus aucun patient (20 sur 21, soit $95,2 \%)$ ne présentait d'ESPT à la fin de la thérapie EMDR. 


\section{Discussion}

Efficacité comparée de l'EMDR et de la TCC dans la phase de suivi du cancer

Le résultat le plus significatif de cette étude est le fait que la plupart des patients traités par l'EMDR dans la phase de suivi du cancer ont pu surmonter leur ESPT après huit séances de thérapie ; au contraire, presque tous les patients dans la même phase de la maladie, mais traités par la TCC, avaient encore un diagnostic d'ESPT un mois après la fin de la psychothérapie.

L'EMDR a significativement réduit les symptômes de stress post-traumatique, mesurés avec la note totale à l'IES-R, la TCC non. Les symptômes intrusifs mesurés par le critère B du CAPS ont significativement baissé tant pour les patients traités par EMDR que pour ceux traités par TCC, bien que, dans le groupe EMDR, la baisse soit plus marquée : le groupe de patients traités par l'EMDR avait, à la fin de la thérapie, à la fois des notes plus basses à l'IES-R et à la sous-échelle des symptômes intrusifs du CAPS que le groupe TCC.

L'anxiété, la dépression et les réactions psychophysiologiques se sont améliorées dans les deux groupes, montrant que les deux types de thérapie sont efficaces sur ces symptômes avec un nombre limité de séances.

Efficacité de l'EMDR au cours des deux phases distinctes de la maladie (traitement médical actif/suivi)

L'EMDR a été efficace tant dans la phase de traitement actif du cancer que dans la phase de suivi de la maladie. Tous les patients ont montré un progrès clinique sur le plan de l'ESPT, l'anxiété, la dépression et les réactions psychophysiologiques.

\section{Limites et conclusions}

Cette étude souffre également de plusieurs limites. En premier lieu, le nombre des patients traités par EMDR ou par TCC n'est pas élevé.

Une autre limite réside dans le fait qu'il n'y a pas eu de vérifications de fidélité pendant les séances de thérapie. Enfin, tous les patients de chaque groupe ont eu le même thérapeute, ce qui laisse la possibilité que les résultats s'expliqueraient par des différences de compétences cliniques sur des variables non spécifiques, par exemple le développement d'une alliance thérapeutique ou d'autres facteurs encore.

Il pourrait être intéressant de mener une autre recherche pour aborder certaines des questions auxquelles cette étude n’a pas pleinement répondu et pour corriger les limites de cette dernière : par exemple, un nombre plus élevé de patients traités et davantage de thérapeutes dans chaque groupe de thérapie. Il faudrait aussi introduire des vérifications de fidélité. Enfin, il faudrait assurer un suivi d'au moins six mois après la fin de la thérapie pour montrer la stabilité des effets de celle-ci dans les différentes conditions des patients.

Si nos résultats ne peuvent être considérés que comme préliminaires, cette étude pilote suggère cependant que, chez des patients atteints de cancer en phase de suivi, l'EMDR a un avantage sur la TCC : elle élimine le diagnostic d'ESPT. L'EMDR a été significativement plus efficace que la TCC pour réduire les notes à l'IES-R et à la sous-échelle des symptômes intrusifs du CAPS, alors que les deux formes de thérapie apparaissent également efficaces sur l'anxiété-trait, la dépression et les réactions psycho-physiologiques.

Notre étude donne à penser que l'EMDR pourrait être une thérapie valable pour les patients atteints de cancer et ayant un diagnostic d'ESPT, tant dans la phase de traitement médical actif que dans la phase de suivi. Ces résultats positifs dans les deux conditions de traitement montrent qu'il est d'une importance capitale que les patients atteints de cancer aient accès à un soutien psychologique et à des formes de thérapie dont on a prouvé l'efficacité, pour leur permettre de gérer les nombreuses difficultés de l'adaptation au statut de rescapé du cancer, et pour les aider à entrer dans un processus positif de résilience psychologique.

Nos données peuvent contribuer à de meilleures connaissances, chez les médecins, des symptômes psychologiques qui peuvent être provoqués par le cancer et qui pourraient être des précurseurs d'un diagnostic d'ESPT. Avec ces informations, ils peuvent adresser rapidement le patient en psychothérapie.

Comme cette étude le montre, une psychothérapie EMDR ou TCC peut être efficace, même en un nombre limité de séances. Pour confirmer et élargir les résultats de cette investigation préliminaire, il faudrait de nouvelles recherches portant sur un échantillon plus grand de patients.

Pour conclure, notre étude suggère que l'EMDR et la TCC sont toutes deux efficaces pour traiter de nombreux symptômes psychologiques chez les patients atteints de cancer, mais nos résultats donnent à penser que l'EMDR pourrait être une forme de thérapie plus efficace que la TCC pour les patients ayant un diagnostic d'ESPT, en particulier pour les symptômes intrusifs, à la fois lors de la phase active du traitement médical et dans la phase de suivi de la maladie. 


\section{Références}

Andersen, B. L., Kiecolt-Glaser, J. K. \& Glaser, R. (1994). A biobehavioral model of cancer stress and disease course. The American Psychologist, 49, 389-404.

American Psychiatric Association. (1987). Diagnostic and statistical manual of mental disorders ( $3^{\mathrm{e}}$ éd., éd. révisée). Washington, DC: Auteur.

American Psychiatric Association. (2000). Diagnostic and statistical manual of mental disorders ( $4^{e}$ éd., texte révisé). Washington, DC: Auteur.

Arabia, E., Manca, M. L. \& Solomon, R. M. (2011). EMDR for survivors of life-threatening cardiac events: Results of a pilot study. Journal of EMDR Practice and Research, 5, 2-13.

Beck, A. T., Rush, A. J., Shaw, B. F. \& Emery, G. (1979). Cognitive therapy of depression. New York, NY: Guilford Press.

Beck, A. T. \& Steer, R. A. (1993). Manual for the Revised Beck Depression Inventory. San Antonio, TX: Psychological Corporation.

Bisson, J. I., Ehlers, A., Matthews, R., Pilling, S., Richards, D. \& Turner, S. (2007). Psychological treatments for chronic post-traumatic stress disorder. Systematic review and meta- analysis. The British Journal of Psychiatry: The Journal of Mental Science, 190, 97-104.

Blake, D. D., Weathers, F. W., Nagy, L. M., Kaloupek, D. G., Gusman, F. D., Charney, D. S. \& Keane, T. M. (1995). The development of a Clinician-Administered PTSD Scale. Journal of Traumatic Stress, 8, 75-90.

Bruce, M. (2006). A systematic and conceptual review of posttraumatic stress in childhood cancer survivors and their parents. Clinical Psychology Review, 26, 233-256.

Butler, L. D., Koopman, C., Classen, C. \& Spiegel, D. (1999). Traumatic stress, life events, and emotional support in women with metastatic breast cancer: Cancerrelated traumatic stress symptoms associated with past and current stressors. Health Psychology, 18, 555-560.

Castrogiovanni, P. \& Traverso, S. (2006). Per una definizione della traumaticità dell'evento. Nòos, 44, 123-149.

Chemtob, C. M., Tolin, D. F., Van der Kolk, B. A. \& Pitman, R. K. (2000). Eye movement desensitization and reprocessing. In E. B. Foa, T. M. Keane, \& M. J. Friedman (Eds.), Effective treatments for PTSD: Practice guidelines from the International Society for Traumatic Stress Studies (pp. 139-154). New York, NY: Guilford Press.

Cordova, M. J., Studts, J. L., Hann, D. M., Jacobsen, P. B. \& Andrykowski, M. A. (2000). Symptom structure of PTSD following breast cancer. Journal of Traumatic Stress, 13, 301-319.

DuHamel, K. N., Ostrof, J., Ashman, T., Winkel, G., Mundy, E. A., Keane, T. M., ... Redd, W. (2004). Construct validity of the posttraumatic stress disorder checklist in cancer survivors: Analyses based on two samples. Psychological Assessment, 16, 255-266.

Ellis, A. (1994). Reason and emotion in psychotherapy, revised and updated. Secaucus, NJ: Carol Publishing Group.
Foa, B. E., Keane, T. M., Friedman, M. J. \& Cohen, J. A. (2008). Effective treatments for PTSD: Practice guidelines from the International Society for Traumatic Stress Studies ( $2^{\mathrm{e}}$ éd.). New York, NY: Guilford Press.

Foa, E. B. \& Tolin, D. F. (2000). Comparison of the PTSD symptom scale-interview version and the Clinician-Administered PTSD Scale. Journal of Traumatic Stress, 13, 181-191.

Friedberg, F. (2004). Eye movement desensitization in fibromyalgia: A pilot study. Complementary Therapies in Nursing and Midwifery, 10, 245-249.

Grant, M. \& Threlfo, C. (2002). EMDR in the treatment of chronic pain. Journal of Clinical Psychology, 58, 1505-1520.

Jackson, J. C., Hart, R. P., Gordon, S. M., Hopkins, R. O., Girard, T. D. \& Ely, E. W. (2007). Post-traumatic stress disorder and post-traumatic stress symptoms following critical illness in medical intensive care unit patients: Assessing the magnitude of the problem. Critical Care, 11, R27.

Mehnert, A., \& Koch, U. (2007). Prevalence of acute and post-traumatic stress disorder and comorbid mental disorders in breast cancer patients during primary cancer care: A prospective study. Psycho-oncology, 16, 181-188.

Morasso, G. (2002). Nuove prospettive in psico-oncologia. In Formazione, psicologia, psicoterapia, psichiatria (pp. 2). Roma, Italy: Grin SRL.

National Cancer Institute, at the United States National Institutes of Health. (2012a). Post-traumatic Stress Disorder (PDQ®) Overview [health professional version]. Récupéré sur http://www.cancer.gov/cancertopics/ $\mathrm{pdq} /$ supportivecare/post-traumatic-stress / Health Professional

National Cancer Institute, at the United States National Institutes of Health. (2012b). Post-traumatic Stress Disorder (PDQ®) Overview [patient version]. Récupéré sur http:/ / www.cancer.gov/cancertopics / pdq/supportivecare/ post-traumatic-stress / Patient/ page2Duke

Onofri, A. (2012). EMDR in psichiatria. Introduzione al supplemento. Rivista di Psichiatria, 47 (Suppl. 2), 1-3.

Pancheri, P., Chiari, G. \& Michielin, P. (1985). Questionario Psicofisiologico, Forma ridotta. Florence, Italy: Organizzazioni Speciali.

Rothbaum, B. O., Astin, M. C. \& Marsteller, F. (2005). Prolonged exposure versus eye movement desensitization and reprocessing (EMDR) for PTSD rape victims. Journal of Traumatic Stress, 18, 607-616.

Schneider, J., Hofmann, A., Rost, C. \& Shapiro, F. (2008). EMDR in the treatment of chronic phantom limb pain. Pain Medicine, 9, 76-82.

Shapiro, F. (1995). Eye movement desensitization and reprocessing: Basic principles, protocols and procedures. New York, NY: Guilford Press.

Shapiro, F. (2001). Eye movement desensitization and reprocessing: Basic principles, protocols and procedures ( $2^{\mathrm{e}}$ éd.). New York, NY: Guildford Press.

Smith, S. K., Zimmerman, S., Williams, C. S., Benecha, H., Abernethy, A. P., Mayer, D. K., . . . Ganz, P. A. (2011). Posttraumatic stress symptoms in long-term 
non-Hodgkin's lymphoma survivors: Does time heal? Journal of Clinical Oncology, 29, 4526-4533.

Spielberger, C. D., Gorsuch, R. L. \& Lushene, R. E. (1970). Manual for the State-Trait Anxiety Inventory. Palo Alto, CA: Consulting Psychologists Press.

Taylor, S., Thordarson, D. S., Maxfield, L., Fedoroff, I. C., Lovell, K. \& Ogrodniczuk, J. S. (2003). Comparative efficacy, speed, and adverse effects of three PTSD treatments: Exposure therapy, EMDR, and relaxation training. Journal of Consulting and Clinical Psychology, 71, 330-338.

Weathers, F. W., Keane, T. M. \& Davidson, J. R. (2001). Clinician-Administered PTSD Scale: A review of the first ten years of research. Depression and Anxiety, 13, 132-156.

Weiss, D. S., \& Marmar, C. R. (1997). The Impact of Event Scale-Revised. In J. P. Wilson \& T. M. Keane (Eds.), Assessing psychological trauma and PTSD (pp. 399-411). New York, NY: Guilford Press.

Remerciements. La présente étude a été subventionnée par le Directeur scientifique du National Cancer Institute
(Istituto Regina Elena-Istituto San Gallicano [IRE-ISG]) de Rome, entre 2009 et 2011.

Notre profonde gratitude va aux services de chirurgie digestive, de chirurgie thoracique, au service $\mathrm{B}$ d'oncologie médicale, et à l'UOSD de l'Area di Supporto alla Persona à l'IRE-ISG (Rome) pour nous avoir permis de travailler avec leurs patients et avoir informés ceux-ci de notre étude.

Nous remercions le Dr Diana Giannarelli (IRE-ISG, Rome) qui a traité les données recueillies et en a fait l'analyse statistique.

Les auteurs souhaitent enfin remercier Lesley Pritikin qui a revu le texte.

Toute correspondance concernant cet article doit être adressée à Liuva Capezzani, UOSD Psichiatria-Area di Supporto alla Persona, IRE-ISG (Istituto Fisioterapici Ospitalieri [IFO]), Via Elio Chianesi, 53, 00144 Roma - Italie. Courriel : liuva@libero.it ou capezzani@ifo.it 